\title{
Kennzahlenbasierte Bewertung von RFID-Systemen
}

\author{
Evaluation of RFID Equipment using Performance Figures
}

\author{
Benjamin Emde \\ Jan-Florian Höfinghoff \\ Ludger Overmeyer \\ Institut für Transport- und Automatisierungstechnik \\ Leibniz Universität Hannover
}

D er Beitrag beschreibt ein optimiertes Kennzahlensystem zur Bewertung von RFID-Systemen beim Einsatz in logistischen Prozessen. Das Verfahren bildet die Verhaltensmerkmale eines RFID-Systems in unterschiedlichen Kennzahlen ab. Die neu definierte Kommunikationsbelastbarkeit ermöglicht erstmals eine anwendungsbezogene Bewertung verschiedener RFIDSysteme auf Basis einer Kennzahl. Am Beispiel der Identifikation eines Regallagerplatzes wird die Aussagekraft des Kennzahlensystems dargestellt.

[Schlüsselwörter: Bewertung, Flurförderzeug, Kennzahlen, RFID]

$\mathbf{T}$ his paper describes an optimized performance figure system for the evaluation of RFID-Systems by the adoption in logistic processes. The method describes the characteristics of the RFID-System in different performance figures. A new defined communicationcapacity provided the first time an applied evaluation based on one performance figure. The significance of the performance figure system is demonstrated for the identification of a high-bay rack.

[Keywords: Evaluation, Forklift Truck, Performance Figures, RFID]

\section{Einleitung}

Durch den Einsatz von Flurförderzeugen lassen sich logistische Prozesse flexibel gestalten. Eine entscheidende Rolle spielt hierbei die Identifikation der Ladung (bzw. des Ladungsträgers) und des Lagerplatzes. Hierzu können RFID-Systeme (engl. Radio Frequency Identification) eingesetzt werden, wodurch eine automatische Identifikation erfolgen kann. Im Vergleich zu herkömmlichen Identifikationssystemen, wie z.B. dem Barcode, ermöglicht ein RFID-System eine kontinuierliche Datenspeicherung direkt an der Ladung bzw. dem Ladungsträger. Aufgrund der geringen Größe sowie der benötigten Reichweite werden passive UHF-Transponder (engl. Ultra High Frequency) für die Kennzeichnung von Ladungsträgern und
Lagerplätzen eingesetzt [Deierling.2010]. Durch den Einsatz entsprechender Schreib-/Lesegeräte und Antennen am Gabelträger bzw. den Gabelzinken des Flurförderzeuges, erfolgt die Kommunikation mit den RFID-Transpondern. Das Flurförderzeug dient somit als mobiles Gate zur Datenverarbeitung [Deierling.2010, Jungk.2007].

Zur Bewertung von RFID-System wird häufig die maximale Reichweite herangezogen. Die Reichweite eines RFID-Systems ist jedoch stark abhängig von den Umgebungsbedingungen. Befinden sich Gegenstände aus Metall im Kommunikationsbereich des RFID-Systems, so kann es zu Interferenzeffekten kommen, wodurch eine Reichweitenreduzierung aber auch -erhöhung hervorgerufenen werden kann. Flüssige Komponenten wirken absorbierend auf die elektromagnetischen Wellen, wohingegen Metalle reflektierend wirken. Um diese Einflüsse bei der Ermittlung der maximalen Reichweite zu vermeiden, erfolgen Messungen der Reichweite häufig in Absorptionskammern [Strauß.2009]. Des Weiteren ist die maximale Reichweite durch die Chipempfindlichkeit des RFID-Transponders bestimmt [Nikitin.2006].

Logistische Prozesse stellen jedoch besondere Herausforderungen an ein RFID-System. Bei der Identifikation von Regal- oder Bodenlagerplätzen kommt es zu vermehrten Reflexionen. Ein nicht $\mathrm{zu}$ vernachlässigen Einfluss besitzt der Ladungsträger sowie dessen Beladung. Durch die Beladung eines Flurförderzuges mit einer Gitterbox, kommt es zur Ablenkung der elektromagnetischen Wellen in Richtung Boden [Höfinghoff.2011]. Hierdurch wird die Reichweite des RFID-Systems stark beeinflusst. Ein weiterer Aspekt den es zu berücksichtigen gilt, ist das Übersprechen zwischen benachbarten Ladungsträgern bzw. Lagerplätzen. Um unterschiedliche RFID-Systeme beim Einsatz in logistischen Prozessen gegeneinander bewerten zu können, ist die Aussage über die maximale Reichweite des jeweiligen Systems nicht ausreichend. Jungk hat hierfür ein Kennzahlensystem entwickelt, welches es ermöglicht, ein RFID-System hinsichtlich der geforderten Funktionen zu bewerten [Jungk.2010]. Am Institut für Transport- und Automatisierungstechnik wurde in weiterführenden Untersuchun- 
gen das Kennzahlensystem hinsichtlich der Anwendbarkeit optimiert. Eine wesentliche Neuerung liegt in der Auswertung der Sendeleistung sowie der neu definierten Kommunikationsbelastbarkeit. Im Folgenden wird die optimierte Form dieses Kennzahlensystems vorgestellt.

\section{BASIS DES KENNZAHLENSYSTEMS}

\subsection{KOORDINATENSYSTEM}

Um die Position der Antenne des Schreib/Lesegerätes und des RFID-Transponder beschreiben zu können, wird ein dreidimensionales kartesisches Koordinatensystem zugrunde gelegt. Der Mittelpunkt der Antenne des Schreib-/Lesegerätes wird durch das feststehende Koordinatensystem $\mathrm{x}, \mathrm{y}$ und $\mathrm{z}$ abgebildet (Abbildung 1). Ein zweites, bewegliches Koordinatensystem $\mathrm{x}^{\prime}, \mathrm{y}^{\prime}$ und $\mathrm{z}^{\prime}$ definiert den Mittelpunkt des RFID-Transponders (Abbildung 1). Der vektorielle Abstand $\mathrm{L}$ zwischen der Antenne des Schreib-/Lesegerätes und dem RFID-Transponder lässt sich nach der Gleichung 1 berechnen.

$$
\underline{L}=\left(\begin{array}{l}
x-x^{\prime} \\
y-y^{\prime} \\
z-z^{\prime}
\end{array}\right)
$$

Die Orientierung der beiden Komponenten zueinander wird mittels des Orientierungsvektors $\underline{\mathrm{O}}$ mit der Gleichung 2 beschrieben.

$$
\underline{O}=\left(\begin{array}{c}
\psi-\psi^{\prime} \\
\theta-\theta^{\prime} \\
\phi-\phi^{\prime}
\end{array}\right)
$$

Innerhalb des feststehenden Koordinatensystems lässt sich der Messbereich $\underline{L}_{\mathrm{Mb}}$ definieren. In diesem Messbereich gibt es Messpunkte, an denen eine Kommunikation zwischen dem Schreib-/Lesegerät und RFID-Transponder immer, manchmal oder niemals stattfindet.

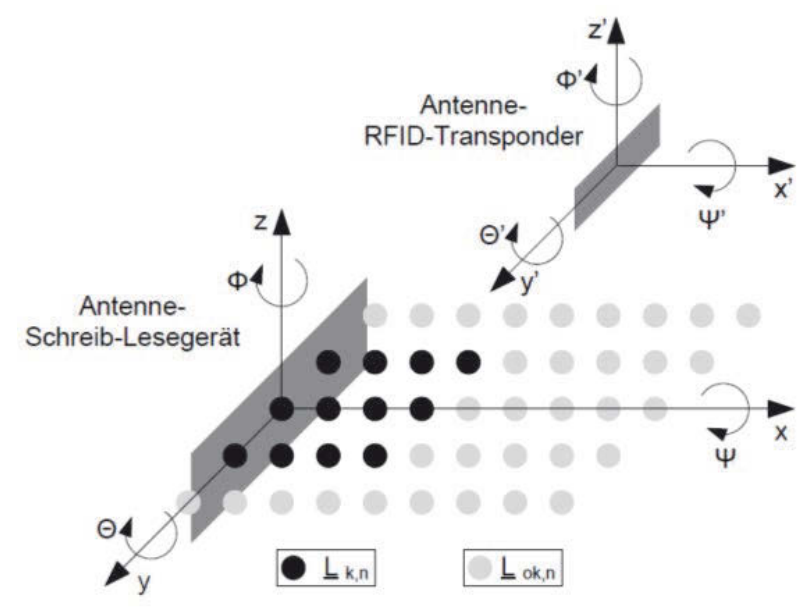

Abbildung 1. Koordinatensystem sowie ein beispielhafter Messbereich mit dem Bereich der sicheren Kommunikation $\underline{L}_{k, n}$ und dem Bereich ohne Kommunikation $\underline{L}_{o k, n}$

$$
\underline{L}_{n}=\underline{n} \cdot d \text { mit } \underline{n}=\left(\begin{array}{l}
n_{x} \\
n_{y} \\
n_{z}
\end{array}\right)
$$

Der Bereich an dem immer eine Kommunikation stattfinden soll, wird dem Bereich der sicheren Kommunikation $\underline{L}_{k, n}$ zugeordnet. Im Bereich ohne Kommunikation $\underline{L}_{o k, n}$ findet keine Kommunikation zwischen Schreib/Lesegerät und RFID-Transponder statt. Die Größe der Bereiche ist vom zu untersuchenden Szenario abhängig. Die Messungen werden inkrementell mit dem Abstand $d$ durchgeführt. Der inkrementelle Abstand $\underline{L}_{n}$ ist nach Gleichung 3 definiert.

\subsection{RELATIVE HÄUFIGKEIT EINER LESUNG}

An jedem Inkrement werden $M$ Messungen durchgeführt und es wird geprüft, ob einen Kommunikation zwischen Schreib-/Lesegerät und RFID-Transponder möglich ist. Das Ergebnis einer Messung wird durch $\mathrm{e}_{\mathrm{m}}\left(\underline{\mathrm{L}}_{\mathrm{n}}\right)$ beschrieben und kann nach Gleichung 4 zwei Zustände annehmen.

$$
e_{m}\left(\underline{L}_{n}\right)= \begin{cases}1, & \text { falls Lesung erfolgreich } \\ 0, & \text { falls Lesung nicht erfolgreich }\end{cases}
$$

Die relative Häufigkeit einer Lesung $p\left(\underline{L}_{n}\right)$ berechnet sich nach Gleichung 5 . Um jedoch ein belastbares Ergebnis zu erhalten, muss eine Messung an einem Inkrement hinreichend oft wiederholt werden $(\mathrm{M} \geq 10)$.

$$
p\left(\underline{L}_{n}\right)=\frac{1}{M} \cdot \sum_{m=0}^{M} e_{m}\left(\underline{L}_{n}\right)
$$

Im Rahmen der Optimierung wurde die Kommunikationsfähigkeit der benachbarten RFID-Transponder (z.B. RFID-Transponder eines benachbarten Regallagerplatzes) untersucht. Hierzu werden an jedem Inkrement $M$ Messungen für die $I$ benachbarten RFID-Transponder durchgeführt. Das Ergebnis einer Messung wird durch $r_{m, i}\left(\underline{L}_{n}\right)$ beschrieben und kann nach Gleichung 6 zwei Zustände annehmen.

$$
r_{m, i}\left(\underline{L}_{n}\right)= \begin{cases}1, & \text { falls Lesung erfolgreich } \\ 0, & \text { falls Lesung nicht erfolgreich }\end{cases}
$$

Die neu definierte relative Häufigkeit einer Lesung der benachbarten RFID-Transponder $t\left(\underline{L}_{n}\right)$ kann mit der Gleichung 7 berechnen werden.

$$
t\left(\underline{L}_{n}\right)=\frac{1}{I \cdot M} \cdot \sum_{i=0}^{I} \sum_{m=0}^{M} r_{m, i}\left(\underline{L}_{n}\right)
$$

\subsection{ERgEBNISÜBERFÜHRUNG IN EINE BINÄRE AUSSAGE}

Aus technischer Sicht interessieren lediglich Inkremente, an denen immer eine Kommunikation zwischen Schreib-/Lesegerät und RFID-Transponder möglich ist $\left(p\left(\underline{L}_{n}\right)=1\right)$ bzw. die Inkremente, an denen keine Kommu- 
nikation möglich ist $\left(\mathrm{p}\left(\underline{\underline{L}}_{n}\right)=0\right)$. Bei der Überführung der relativen Häufigkeit einer Lesung $p\left(\underline{L}_{n}\right)$ in eine binäre Aussage $\rho\left(\underline{L}_{n}\right)$, muss jedoch auch die neu definierte relative Häufigkeit einer Lesung der benachbarten RFIDTransponder $t\left(\underline{L}_{n}\right)$ berücksichtigt werden. Eine sichere Kommunikation $\left(\rho\left(\underline{L}_{n}\right)=1\right)$ liegt dann vor, wenn die relative Häufigkeit einer Lesung $p\left(\underline{L}_{n}\right)=1$ und die relative Häufigkeit einer Lesung der benachbarten RFID-Transponder $\mathrm{t}\left(\underline{\underline{L}}_{\mathrm{n}}\right)=0$ ist. Für andere Konstellationen liegt keine sichere Kommunikation vor $\left(\rho\left(\underline{L}_{n}\right)<1\right.$ und $\left.t\left(\underline{L}_{n}\right)>0\right)$. Gleichung 8 zeigt diese optimierte Ergebnisüberführung.

$$
\rho\left(\underline{L}_{n}\right)= \begin{cases}1, & \text { falls } p\left(\underline{L}_{n}\right)-t\left(\underline{L}_{n}\right)=1 \\ 0, & \text { falls } p\left(\underline{L}_{n}\right)-t\left(\underline{L}_{n}\right)<1\end{cases}
$$

Analog hierzu kann der Bereich ohne Kommunikation durch $\mu\left(\underline{L}_{n}\right)$ beschrieben werden. Ein Inkrement ohne Kommunikation $\left(\mu\left(\underline{L}_{n}\right)=1\right)$ liegt dann vor, wenn die relative Häufigkeit einer Lesung $p\left(\underline{L}_{n}\right)=0$ und die relative Häufigkeit einer Lesung der benachbarten RFID-Transponder $t\left(\underline{\underline{L}}_{n}\right)=0$ ist (Gleichung 9).

$$
\mu\left(\underline{L}_{n}\right)= \begin{cases}1, & \text { falls } p\left(\underline{L}_{n}\right)+t\left(\underline{L}_{n}\right)=0 \\ 0, & \text { falls } p\left(\underline{L}_{n}\right)+t\left(\underline{L}_{n}\right)>0\end{cases}
$$

\subsection{DEFINITION EINER SOLLFUNKTION}

Um die Inkremente bei der Berechnung der Kennzahlen gewichten zu können, werden zwei Soll-Funktionen definiert. Der Bereich der sicheren Kommunikation wird durch die Funktion $\mathrm{f}\left(\underline{\underline{L}}_{\mathrm{n}}\right)$ abgebildet (Gleichung 10). Diese weist den Wert Eins auf, wenn sich das untersuchte Inkrement im Bereich der sicheren Kommunikation befindet.

$$
f\left(\underline{L}_{n}\right)= \begin{cases}1, & \text { falls } \underline{L}_{n} \in \underline{L}_{k, n} \\ 0, & \text { falls } \underline{L}_{n} \in \underline{L}_{o k, n}\end{cases}
$$

Der Bereich ohne Kommunikation wird durch die Funktion $\bar{f}\left(\underline{L}_{n}\right)$ beschrieben (Gleichung 11). Analog zur Funktion $\mathrm{f}\left(\underline{\underline{L}}_{n}\right)$ weist die Funktion $\overline{\mathrm{f}}\left(\underline{\underline{L}}_{n}\right)$ den Wert Eins auf, wenn sich das zu untersuchende Inkrement im Bereich ohne Kommunikation befindet.

$$
\bar{f}\left(\underline{L}_{n}\right)= \begin{cases}0, & \text { falls } \underline{L}_{n} \in \underline{L}_{k, n} \\ 1, & \text { falls } \underline{L}_{n} \in \underline{L}_{o k, n}\end{cases}
$$

\section{KENNZAHLEN}

\subsection{LESEFÄHIGKEIT}

Die Lesefähigkeit LF ermöglicht eine Beurteilung, wie gut ein RFID-Transponder im Bereich der sicheren Kommunikation gelesen werden kann. Hierzu werden in der Gleichung 12 alle Inkremente des Bereichs der sicheren Kommunikation, an denen einen Kommunikation zwischen Schreib-/Lesegerät und RFID-Transponder möglich ist, aufaddiert. Das Optimum liegt dann vor, wenn die Lesefähigkeit LF $=1$ ist.

$$
L F=\frac{\sum_{n_{x, \min }}^{n_{x, \max }} \sum_{n_{y, \min }}^{n_{y, \max }} \sum_{n_{z, \min }}^{n_{z, \max }}\left(f\left(\underline{L}_{n}\right) \cdot \rho\left(\underline{L}_{n}\right)\right)}{\sum_{n_{x, \min }}^{n_{x, \max }} \sum_{n_{y, \min }}^{n_{y, \max }} \sum_{n_{z, \min }}^{n_{z, \max }} f\left(\underline{L}_{n}\right)}
$$

\subsection{SCHREIBFÄHIGKEIT}

Bei Identifikationsprozessen, bei denen der RFIDTransponder beschrieben werden muss, kann die Schreibfähigkeit SF berechnet werden (Gleichung 13). Der Unterschied zur Lesefähigkeit LF besteht darin, dass hierbei nur vom Identifikationsprozess abhängige Inkremente betrachtet werden. Das Optimum liegt dann vor, wenn die Schreibfähigkeit SF = 1 ist.

$$
S F=\frac{\sum_{n_{x}=0}^{1} \sum_{n_{y}=0}^{0} \sum_{n_{z}=0}^{0}\left(f\left(\underline{L}_{n}\right) \cdot \rho\left(\underline{L}_{n}\right)\right)}{\sum_{n_{x}=0}^{1} \sum_{n_{y}=0}^{0} \sum_{n_{z}=0}^{0} f\left(\underline{L}_{n}\right)}
$$

\subsection{KOMMUNIKATIONSUNFÄHIGKEIT}

Der Bereich ohne Kommunikation wird durch die Kommunikationsunfähigkeit KU bewertet. Hierbei werden in der Gleichung 14 alle Inkremente im Bereich ohne Kommunikation aufaddiert, an denen keine Kommunikation zwischen Schreib-/Lesegerät und RFID-Transponder möglich ist. Weist die Kommunikationsunfähigkeit den Wert KU = 1 auf, so ist an keinem Inkrement des Bereichs ohne Kommunikation eine Kommunikation zwischen Schreib-/Lesegerät und RFID-Transponder möglich.

$$
K U=\frac{\sum_{n_{x, \min }}^{n_{x, \max }} \sum_{n_{y, \min }}^{n_{y, \max }} \sum_{n_{z, \min }}^{n_{z, \max }}\left(\bar{f}\left(\underline{L}_{n}\right) \cdot \mu\left(\underline{L}_{n}\right)\right)}{\sum_{n_{x, \min }}^{n_{x, \max }} \sum_{n_{y, \min }}^{n_{y, \max }} \sum_{n_{z, \min }}^{n_{z, \max }} \bar{f}\left(\underline{L}_{n}\right)}
$$

\subsection{ORIENTIERUNGSUNEMPFINDLICHKEIT}

Je nach Anwendungsfall liegen unterschiedliche Orientierungen zwischen Schreib-Lesegerät und RFIDTransponder vor. Eine Palette kann z.B. aus vier Richtungen aufgenommen werden. Da die Orientierung Auswirkungen auf das Systemverhalten besitzt, sind die zuvor genannten Kennzahlen für alle relevanten Orientierungen zu bestimmen. Um die Kennzahlen dennoch leicht auswerten zu können, kann nach der Gleichung 15 der Mittelwert für die unterschiedlichen Orientierungen berechnet werden (hier am Beispiel der Lesefähigkeit).

$$
\overline{L F}=\frac{1}{K} \cdot \sum_{k=0}^{K} L F_{k}
$$

Um eine Aussage treffen zu können, wie stark sich eine Orientierungsänderung im Mittel auf eine Kennzahl auswirkt, wurde von Jungk die Orientierungsempfindlichkeit OE definiert. Für ein gegen Orientierungsänderungunempfindliches RFID-System ergibt sich eine Orientierungsempfindlichkeit von $\mathrm{OE}=0$. Dies ist aus mathematischer Sicht korrekt, ist für den Anwender jedoch nicht anschaulich, da alle anderen Kennzahlen ihr Optimum bei „1“ haben. Als Ersatz wurde daher die Orientierungsunempfindlichkeit OU definiert. Die Orientie- 
rungsunempfindlichkeit der Lesefähigkeit $\mathrm{OU}_{\mathrm{LF}}$ berechnet sich nach der Gleichung 16.

$$
O U_{L F}=1-\left[\left(L F_{1}-\overline{L F}\right)^{2}+\cdots+\left(L F_{K}-\overline{L F}\right)^{2}\right]^{-\frac{1}{2}}
$$

Die gesamte Orientierungsunempfindlichkeit OU in der Gleichung 17 fasst die Orientierungsunempfindlichkeit der einzelnen Kennzahlen zusammen. Im Vergleich zur Berechnung der Orientierungsempfindlichkeit OE nach Jungk, erfolgt die Berechnung der Orientierungsunempfindlichkeit OU mittels Fallunterscheidung. Dies erfolgt nicht aus mathematischen sondern nur aus anwendungsbezogenen Gründen (Eine Orientierungsunempfindlichkeit von $\mathrm{OU}=1$ für eine Lesefähigkeit von $\mathrm{LF}=0$ könnte den Anwender verwirren). Für den Fall, dass keine Kommunikation zwischen Schreib-/Lesegerät und RFIDTransponder möglich ist $(\mathrm{LF}=0)$, wird die Orientierungsunempfindlichkeit entsprechend zu null gesetzt.

$$
O U=\left\{\begin{aligned}
0, & \text { falls } L F & =0 \\
\frac{1}{3}\left(O U_{L F}+O U_{S F}+O U_{K U},\right. & \text { falls } L F & \neq 0
\end{aligned}\right.
$$

\subsection{KOMMUNIKATIONSSICHERHEIT}

Um eine komprimierte Darstellung aller bisher ermittelten Kennzahlen zu erhalten, wurde von Jungk die Gesamtkennzahl Kommunikationssicherheit KS definiert. Diese spiegelt das Kommunikationsverhalten zwischen Schreib-/Lesegerät und RFID-Transponder wieder. Die Berechnung erfolgt durch eine Mittelwertbildung der einzelnen gewichteten Kennzahlen (vgl. Gleichung 18 für den Fall $L F \geq 0,1$ ). Den Bewertungsgewichten $a_{L F}, a_{S F}$, $\mathrm{a}_{\mathrm{KU}}$ und $\mathrm{a}_{\mathrm{OU}}$ kann ein Wert zwischen $0 . .1$ zugewiesen werden. Es gibt für diese Gewichte jedoch keine Erfahrungswerte, sodass bisher stets der Wert Eins oder Null gewählt wurde. Wechselwirkungen zwischen den einzelnen Kennzahlen lassen sich mit diesem Ansatz jedoch nicht abbilden.

Die Grundvoraussetzung für eine sichere Kommunikation ist jedoch, dass eine Kommunikation stattfindet (LF > 0). Daher erfolgt die Berechnung der optimierten Kommunikationssicherheit in der Gleichung 18 mittels Fallunterscheidung. Hierbei ist die Kommunikationssicherheit gleich der Lesefähigkeit, solange die Lesefähigkeit $\mathrm{LF}<0,1$ ist. Hierdurch wird vermieden, dass bei einer schlechten Lesefähigkeit (LF $<0,1$ ) die Kommunikationssicherheit durch ein gute Kommunikationsunfähigkeit bzw. Orientierungsunempfindlichkeit positiv beeinflusst wird. Der optimale Fall liegt dann vor, wenn die Kommunikationssicherheit KS = 1 ist, also alle Kennzahlen ihr Maximum erreicht haben.

$$
K S=\left\{\begin{array}{cr}
L F & \text {, falls } L F<0,1 \\
\frac{L F \cdot a_{L F}+S F \cdot a_{S F}+K U \cdot a_{K U}+O U \cdot a_{O U}}{a_{L F}+a_{S F}+a_{K U}+a_{O U}}, & \text { falls } L F \geq 0,1
\end{array}\right.
$$

Die einzelnen Kennzahlen stehen in Wechselwirkung zueinander. Um diese Wechselwirkung bei der Berech- nung der Kommunikationssicherheit abbilden zu können, werden die Kennzahlen im Rahmen der Optimierung durch neu definierte Bewertungsvariablen $\mathrm{a}_{\mathrm{LF}}, \mathrm{a}_{\mathrm{SF}}, \mathrm{a}_{\mathrm{KU}}$ und $\mathrm{a}_{\mathrm{OU}}$ (Gleichung 19 bis 22) miteinander verknüpft. Soll eine Kennzahl bei der Berechnung der Kommunikationssicherheit nicht berücksichtigt werden, so kann die Kennzahl sowie die entsprechende Bewertungsvariable zu null gesetzt werden.

$$
\begin{aligned}
& a_{L F}=e^{(S F+K U+O U)} \\
& a_{S F}=e^{(L F+K U+O U)} \\
& a_{K U}=e^{(L F+S F+O U)} \\
& a_{O U}=e^{(L F+S F+K U)}
\end{aligned}
$$

\subsection{EINFLUSS DER SENDELEISTUNG}

Bei dem Kennzahlensystem nach Jungk werden die Kennzahlen für eine definierte Sendeleistung ermittelt. Die am Institut für Transport- und Automatisierungstechnik durchgeführten Versuche wurden mit einem Leistungssweep (schrittweise Erhöhung der Sendeleistung) durchgeführt. Hierdurch kann die minimal benötigte Sendeleistung ermittelt werden, die nötig ist, um an einem Messpunkt eine Kommunikation zwischen Schreib/Lesegerät und RFID-Transponder zu ermöglichen. Erstmalig ist es somit möglich, die Kennzahlen als Funktion der Sendeleistung aufzutragen. Aufgrund der Übersichtlichkeit wird jedoch darauf verzichtet, die Kennzahlen als Funktion der Sendeleistung zu kennzeichnen. Die im Folgenden neu definierte optimale Sendeleistung (Kapitel 3.6) und Kommunikationsbelastbarkeit (Kapitel 3.7) lassen sich erst auf Basis dieser Sendeleistungsinformationen bestimmen.

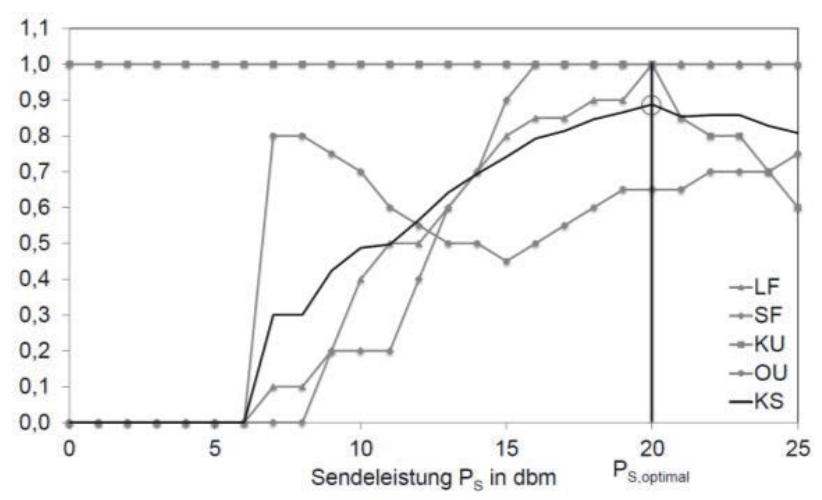

Abbildung 2. Beispielhafte Kennzahlen als Funktion der Sendeleistung

Abbildung 2 zeigt einen beispielhaften Kennzahlenverlauf. Die Sendeleistung, bei der die maximale Kommunikationssicherheit auftritt, wird als die optimale Sendeleistung $\mathrm{P}_{\mathrm{S} \text {,optimal }}$ bezeichnet. Die Bewertung unterschiedlicher RFID-Systeme erfolgt bei dem optimierten Kennzahlensystem stets bei der optimalen Sendeleistung. Somit kann sichergestellt werden, dass jedes 
RFID-System unter optimalen Sendeleistungsbedingungen verglichen wird.

\subsection{KOMMUNIKATIONSBELASTBARKEIT}

In der Anwendung werden die Kennzahlen für die optimale Sendeleistung $\mathrm{P}_{\mathrm{S} \text {,optimal }}$ ermittelt. Der Anwender kann anhand der Werte der einzelnen Kennzahlen unterschiedliche RFID-Systeme miteinander vergleichen. Die Information über die Abhängigkeit der Kennzahlen im Bezug auf die Sendeleistung $\mathrm{P}_{\mathrm{S}}$ geht hierbei jedoch verloren. Um diese Information dem Anwender zu veranschaulichen, wird im Folgenden die Kommunikationsbelastbarkeit KB definiert. Als Basis wird hierfür die Gesamtkennzahl Kommunikationssicherheit KS herangezogen, die sämtliche Kennzahlen zusammenfasst.

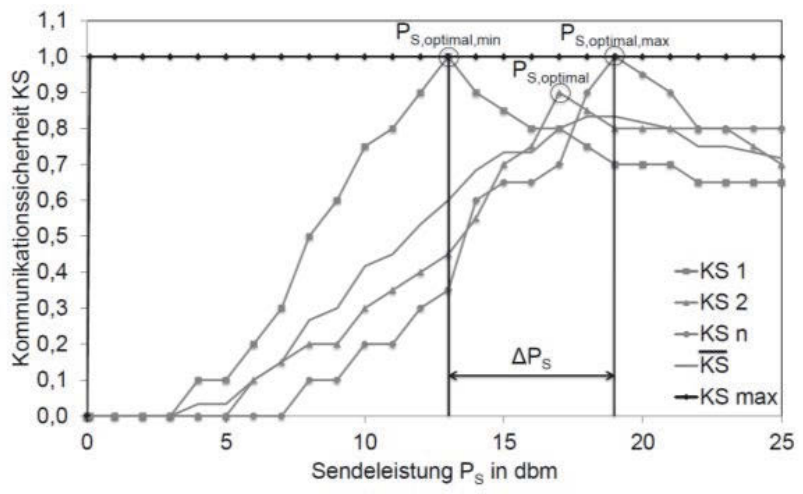

Abbildung 3. Beispielhafte Kommunikationssicherheit als Funktion der Sendeleistung für ein RFID-System (Transponder und Schreib-/Lesegerät)

Betrachtet man beispielsweise die Identifikation eines Regallagerplatzes, so kann zum einen unterschiedliche Beladung (z.B. Metall, Flüssigkeiten, Kunststoff, etc.) aber auch der Lagerprozess (z.B. Ein- bzw. Auslagern eines Ladungsträgers) die Eigenschaften eines RFIDSystems beeinflussen. In der Praxis müssen für alle relevanten Konstellationen (Variation Beladung und Lagerprozess) Messungen durchgeführt werden, um eine praxisnahe Bewertung zu ermöglichen. Abbildung 3 zeigt den Verlauf der Kommunikationssicherheit $\mathrm{KS}_{1}$ bis $\mathrm{KS}_{\mathrm{n}}$ für einen RFID-Transponder (z.B. für eine Regallageridentifikation). Mittels der Gleichung 23 kann die mittlere Kommunikationssicherheit $\overline{K S}\left(\mathrm{P}_{\mathrm{S}}\right)$ berechnet werden. Hierbei erfolgt die Mittelwertbildung über den gesamten Funktionsverlauf bis zur maximal verwendeten Sendeleistung.

$$
\overline{K S}\left(P_{S}\right)=\frac{1}{K} \cdot \sum_{k=0}^{K} K S_{k}\left(P_{S}\right)
$$

Die Fläche unterhalb der mittleren Kommunikationssicherheit $\overline{\mathrm{KS}}\left(\mathrm{P}_{\mathrm{S}}\right)$ beschreibt das Verhalten zwischen der Kommunikationssicherheit und der Sendeleistung. Um eine Normierung vornehmen zu können, wird die maximale Kommunikationssicherheit $\mathrm{KS}_{\max }$ aus Abbildung 3 heran- gezogen. Hierbei weist die Kommunikationssicherheit bereits für $\mathrm{P}_{\mathrm{S}} \rightarrow 0$ einen Wert von $\mathrm{KS}=1$ auf und bleibt für steigende Sendeleistungen konstant. Dieser Verlauf ist aus technischer Sicht unerreichbar, ermöglicht jedoch eine einheitliche Normierung der mittleren Kommunikationssicherheit $\overline{K S}\left(\mathrm{P}_{\mathrm{S}}\right)$. Die Berechnung der Kommunikationsbelastbarkeit erfolgt nach Gleichung 24.

$$
K B_{\left[\Delta P_{S}\right]}=\frac{1}{P_{S, \max }} \cdot \int_{0}^{P_{S, \max }} \overline{K S}\left(P_{S}\right) d P_{S}
$$

Des Weiteren enthält die Kommunikationsbelastbarkeit $\mathrm{KB}$ den optimalen Leistungsbereich $\Delta \mathrm{P}_{\mathrm{S}}$ als Index. Dieser Index gibt an, in welchem Abstand sich die optimalen Sendeleistungen $\mathrm{P}_{\mathrm{S} \text {,optimal }}$ der verschiedenen Kommunikationssicherheiten $\mathrm{KS}_{1}$ bis $\mathrm{KS}_{\mathrm{n}}$ befinden (Gleichung 25). Der optimale Leistungsbereich $\Delta \mathrm{P}_{\mathrm{S}}$ ist somit ein $\mathrm{Ma}$ dafür, wie stark sich wechselnde Umgebungsbedingungen (z.B. Beladungen bzw. Lagerprozesse) auf das RFIDSystem auswirken.

$$
\Delta P_{S}=P_{S, o p t i m a l, \text { max }}-P_{S, o p t i m a l, m i n}
$$

In der Anwendung des Kennzahlensystems wird im ersten Schritt die Kommunikationsbelastbarkeit der einzelnen RFID-Systeme verglichen und die Varianten mit den höchsten Werten für $\mathrm{KB}$ ausgewählt $(\mathrm{KB} \rightarrow 1$ entspricht einem geringen Sendeleistungsbedarf). Im zweiten Schritt kann der optimale Leistungsbereich dieser RFIDSysteme betrachtet werden. Das System mit dem kleinsten $\Delta \mathrm{P}_{\mathrm{S}}$ stellt das Optimum dar.

\section{BEISPIEL: REGALLAGERIDENTIFIKATION}

\subsection{SCHREIB-/LESEGERÄT / RFID-TRANSPONDER}

Als Schreib-/Lesegerät wurde der UDL100 ${ }^{\mathrm{TM}}$ der Firma deister electronic GmbH eingesetzt. Hierbei handelt es sich um ein UHF Schreib-Lesegerät mit einer maximale Sendeleistung von $27 \mathrm{dBm}$ E.R.P. (engl. effective radiated power) und dem Transponderprotokoll ISO 180006 C (EPC Class1 Gen2). Die Montage am Gabelträger sowie die relevanten Maße sind der Abbildung $4 \mathrm{zu}$ entnehmen. Für die Versuche wurden passive UHF-RFIDTransponder eingesetzt. Tabelle 1 zeigt die Anwendungstypen, die Reichweiten (im Freiraum) und die Abmessungen der getesteten kommerziellen RFID-Transponder.

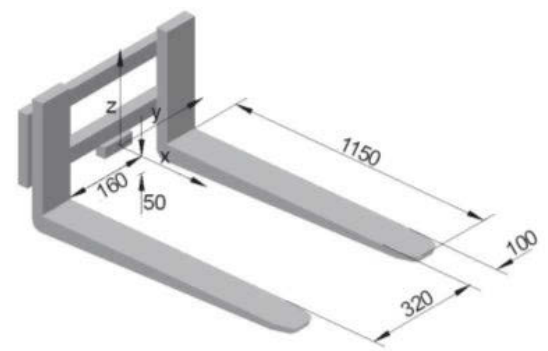

Abbildung 4. Gabelträger mit den relevanten Maßangaben 
Tabelle 1. Daten der eingesetzten Transponder

\begin{tabular}{lll}
\hline $\begin{array}{l}\text { Transponder Nr. } \\
\text { Anwendungstyp }\end{array}$ & $\begin{array}{l}\text { Reichweite in } \\
\text { (Freiraum) }\end{array}$ & $\begin{array}{l}\text { Abmessungen in } \\
\mathrm{mm}\end{array}$ \\
\hline \hline 1/Metall & 5 & $158 \times 22 \times 18$ \\
\hline 2/Flüssigkeit & $>2,5$ & $123 \times 30 \times 8$ \\
\hline 3/Flüssigkeit & $>4,5$ & $165 \times 51 \times 2,6$ \\
\hline 4/Verpackung & 4 & $92 \times 24 \times 0,2$ \\
\hline 5/Metall & $3-4$ & $27 \times 27 \times 5,5$ \\
\hline 5/Metall & 5 & $52 \times 48 \times 10$ \\
\hline
\end{tabular}

\subsection{Definition DES MeSSBEREICHES}

Abbildung 5 zeigt den schematischen Versuchsaufbau für die Identifikation eines Regallagerplatzes. Im oberen Teil des Bildes ist das Regallager mit drei Ladungsträgern zu sehen. Die Montage der RFID-Transponder auf dem Regalprofil erfolgte mit den angegebenen Maßangaben. Der untere Teil des Bildes zeigt die Draufsicht auf das Regalprofil sowie die Gabelzinken. In Tabelle 2 sind die Messbereiche der einzelnen Identifikationsszenarien sowie die Abstände der einzelnen Messpunkte zueinander zu sehen. Der Messbereich ist zum einem von der Beladung (Palette und Gitterbox) und zum anderem vom Lagerprozess (Ein- und Auslagern) abhängig.

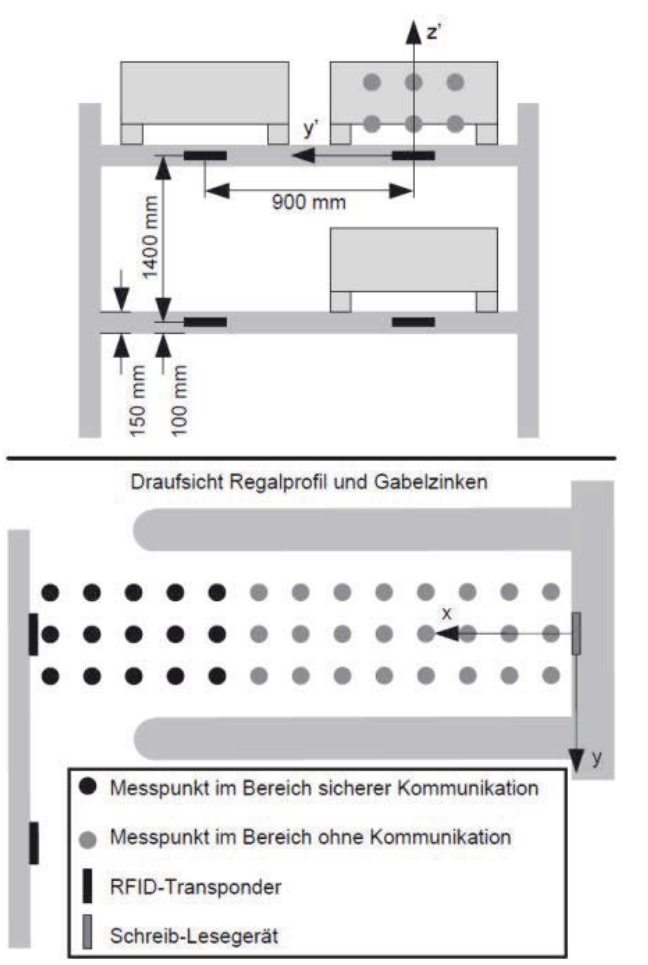

Abbildung 5. Schematischer Versuchsaufbau des Regallagers
Tabelle 2. Messbereich eines Regallagerplatzes

\begin{tabular}{lc}
\hline \multicolumn{1}{c}{ Szenario } & Messbereich \\
\hline \hline Palette/Auslagern & $x=0 \mathrm{~mm}$ bis $1200 \mathrm{~mm}$, alle $100 \mathrm{~mm}$ \\
$y=-40 \mathrm{~mm}, 0 \mathrm{~mm}, 40 \mathrm{~mm}$ \\
$z=0 \mathrm{~mm}$
\end{tabular}

\subsection{VERSUCHSERgEBNISSE}

Die ermittelten Kennzahlen Lesefähigkeit LF, Kommunikationsunfähigkeit KU, Kommunikationssicherheit KS sowie die optimale Sendeleistung $\mathrm{P}_{\mathrm{S} \text {,optimal }}$ sind für den Einlagerprozess in der Tabelle 3 zu sehen. Im Folgenden werden jedoch nur die Kommunikationssicherheit KS und die optimale Sendeleistung $\mathrm{P}_{\mathrm{S} \text {,optimal }}$ betrachtet. Es ist zu erkennen, dass die RFID-Transponder 1, 2 und 6 eine relativ konstante Kommunikationssicherheit KS für unterschiedliche Beladungen aufweisen. Anhand der optimalen Sendeleistung $\mathrm{P}_{\mathrm{S} \text {,optimal }}$ ist zu erkennen, dass bei einer Beladung mit einer Gitterbox eine höher Sendeleistung erforderlich ist. Dies lässt sich dadurch begründen, dass die elektromagnetischen Wellen durch die Gitterbox in Richtung Boden reflektiert werden, wodurch eine Reichweitenreduzierung auftritt. Bei dem RFID-Transponder 4 handelt es sich um einen Labeltransponder, der direkt auf das Regalprofil geklebt wurde. Da hierbei die Transponderantenne von der Metalloberfläche des Regalprofiles kurzgeschlossen wird, kann der Transponder keine Energie aufnehmen - eine Kommunikation ist nicht möglich. Diese Tatsache spiegelt sich entsprechend in der Kommunikationssicherheit KS wieder. Die RFID-Transponder 3 und 5 zeigen bei der Beladung durch eine Gitterbox einen deutlichen Einbruch in der Kommunikationssicherheit KS und weisen einen erhöhten Sendeleistungsbedarf auf. 
Tabelle 3. Kennzahlen der Regallageridentifikation/Einlagern

\begin{tabular}{|c|c|c|c|c|}
\hline $\begin{array}{l}\text { Transponder Nr. } \\
\text { Beladung }\end{array}$ & $L F$ & $K U$ & $K S$ & $\boldsymbol{P}_{S, \text { optimal }}$ \\
\hline 1/Palette & 0,83 & 0,83 & 0,83 & $17 \mathrm{dBm}$ \\
\hline 1/Gitterbox & 0,97 & 1,00 & 0,98 & $22 \mathrm{dBm}$ \\
\hline 1/Palette+Wasser & 0,80 & 0,92 & 0,86 & $15 \mathrm{dBm}$ \\
\hline 2/Palette & 0,90 & 0,82 & 0,86 & $19 \mathrm{dBm}$ \\
\hline 2/Gitterbox & 0,97 & 0,99 & 0,98 & $24 \mathrm{dBm}$ \\
\hline 2/Palette+Wasser & 0,90 & 0,93 & 0,91 & $16 \mathrm{dBm}$ \\
\hline 3/Palette & 0,73 & 0,97 & 0,84 & $27 \mathrm{dBm}$ \\
\hline 3/Gitterbox & 0,03 & 1,00 & 0,30 & $26 \mathrm{dBm}$ \\
\hline 3/Palette+Wasser & 0,47 & 1,00 & 0,66 & $27 \mathrm{dBm}$ \\
\hline 4/Palette & 0,00 & 1,00 & 0,00 & $-d B m$ \\
\hline 4/Gitterbox & 0,00 & 1,00 & 0,00 & $-d B m$ \\
\hline 4/Palette+Wasser & 0,00 & 1,00 & 0,00 & $-d B m$ \\
\hline 5/Palette & 0,87 & 0,82 & 0,84 & $27 \mathrm{dBm}$ \\
\hline 5/Gitterbox & 0,33 & 1,00 & 0,56 & $27 \mathrm{dBm}$ \\
\hline 5/Palette+Wasser & 0,83 & 0,86 & 0,85 & $26 \mathrm{dBm}$ \\
\hline 6/Palette & 0,90 & 0,87 & 0,89 & $20 \mathrm{dBm}$ \\
\hline 6/Gitterbox & 0,97 & 0,95 & 0,96 & $25 \mathrm{dBm}$ \\
\hline 6/Palette+Wasser & 0,87 & 0,97 & 0,91 & $19 \mathrm{dBm}$ \\
\hline
\end{tabular}

In der Tabelle 4 sind die ermittelten Kennzahlen für den Auslagerprozess zu sehen. Die RFID-Transponder 1, 2, 5 und 6 zeigen eine hohe und annähernd konstante Kommunikationssicherheit KS. Es fällt auf, dass die optimale Sendeleistung $\mathrm{P}_{\mathrm{S} \text {,optimal }}$ beim Auslagern einer Gitterbox tendenziell geringer ist, als beim Auslagern einer Palette bzw. einer mit Wasser beladenen Palette. Dies lässt sich dadurch begründen, dass sich zwischen dem Hubmast des Flurförderzeugs und der Gitterbox eine stehende Welle ausbilden kann, woraus ein reduzierter Sendeleistungsbedarf resultiert. Der RFID-Transponder 3 zeigt einen erhöhten Sendeleistungsbedarf sowie eine starken Einbruch der Kommunikationssicherheit KS für die Beladung mit einer Gitterbox und einer mit Wasser beladenen Palette. Der RFID-Transponder 4 ermöglicht keine Kommunikation.

Beim Vergleich der Tabelle 3 und 4 erkennt man, dass zum einen der Lagerprozess und zum anderen die Beladung einen signifikanten Einfluss auf den Sendeleistungsbedarf und somit auch auf die Kennzahlen haben. Die Beladung mit metallischen Gegenständen kann je nach Lagerprozess zu einer Verbesserung oder zu einerVerschlechterung der Systemeigenschaften führen.

Zusammengefasst werden die Ergebnisse des Einund Auslagerprozesses durch die Kommunikationsbelastbarkeit, die in der Tabelle $5 \mathrm{zu}$ sehen ist. Hierbei zeigen die RFID-Transponder 1 und 2 die größte Kommunikationsbelastbarkeit. Der optimale Leistungsbereich beträgt $\mathrm{P}_{\mathrm{S}}=12 \mathrm{dBm}$ bzw. $\mathrm{P}_{\mathrm{S}}=11 \mathrm{dBm}$. Diese RFID-Transponder stellen somit das Optimum der untersuchen RFID-
Transponder dar. Der RFID-Transponder 6 weist neben den RFID-Transponder 1 und 2 mit 45\% ebenfalls eine hohe Kommunikationsbelastbarkeit auf. Diese ist jedoch geringer, da dieser RFID-Transponder einen höheren Sendeleistungsbedarf als die RFID-Transponder 1 und 2 aufweist. Die RFID-Transponder 3, 4 und 5 zeigen das schlechteste Verhalten. Mithilfe der Kommunikationsbelastbarkeit kann somit zusammengefasst die gleiche Aussage über die Ergebnisse der Tests getroffen werden, wie dies auch Tabelle 3 und 4 liefern würden.

Tabelle 4. Kennzahlen der Regallageridentifikation/Auslagern

\begin{tabular}{|c|c|c|c|c|}
\hline $\begin{array}{l}\text { Transponder } \mathrm{Nr} \text {. } \\
\text { Beladung }\end{array}$ & $L F$ & $K U$ & $K S$ & $\boldsymbol{P}_{\text {S,optimal }}$ \\
\hline 1/Palette & 0,73 & 0,95 & 0,83 & $13 \mathrm{dBm}$ \\
\hline 1/Gitterbox & 0,93 & 1,00 & 0,97 & $10 \mathrm{dBm}$ \\
\hline 1/Palette+Wasser & 0,93 & 0,81 & 0,87 & $15 \mathrm{dBm}$ \\
\hline 2/Palette & 0,93 & 0,90 & 0,92 & $15 \mathrm{dBm}$ \\
\hline 2/Gitterbox & 1,00 & 0,86 & 0,93 & $13 \mathrm{dBm}$ \\
\hline 2/Palette+Wasser & 1,00 & 0,95 & 0,98 & $14 \mathrm{dBm}$ \\
\hline 3/Palette & 1,00 & 0,94 & 0,97 & $26 \mathrm{dBm}$ \\
\hline 3/Gitterbox & 0,60 & 1,00 & 0,76 & $26 \mathrm{dBm}$ \\
\hline 3/Palette+Wasser & 0,53 & 1,00 & 0,71 & $25 \mathrm{dBm}$ \\
\hline 4/Palette & 0,00 & 1,00 & 0,00 & $-d B m$ \\
\hline 4/Gitterbox & 0,00 & 1,00 & 0,00 & $-d B m$ \\
\hline 4/Palette +Wasser & 0,00 & 1,00 & 0,00 & $-d B m$ \\
\hline 5/Palette & 1,00 & 0,83 & 0,91 & $25 \mathrm{dBm}$ \\
\hline 5/Gitterbox & 0,80 & 0,84 & 0,82 & $21 \mathrm{dBm}$ \\
\hline 5/Palette +Wasser & 0,93 & 0,79 & 0,86 & $24 \mathrm{dBm}$ \\
\hline 6/Palette & 1,00 & 0,89 & 0,94 & $18 \mathrm{dBm}$ \\
\hline 6/Gitterbox & 0,93 & 0,94 & 0,93 & $17 \mathrm{dBm}$ \\
\hline 6/Palette+Wasser & 1,00 & 0,86 & 0,92 & $20 \mathrm{dBm}$ \\
\hline
\end{tabular}

Tabelle 5. Kommunikationsbelastbarkeit

\begin{tabular}{cc}
\hline Transponder Nr. & $K B_{[\Delta P s]}$ \\
\hline \hline 1 & $0,50_{[12 \mathrm{dBm}]}$ \\
\hline 2 & $0,52_{[11 \mathrm{dBm}]}$ \\
\hline 3 & $0,12_{[2 \mathrm{dBm}]}$ \\
\hline 4 & $0,00_{[-\mathrm{dBm}]}$ \\
\hline 5 & $0,28_{[6 \mathrm{dBm}]}$ \\
\hline 6 & $0,45_{[8 \mathrm{dBm}]}$ \\
\hline
\end{tabular}

\section{ZUSAMMENFASSUNG}

Das überarbeitete Kennzahlensystem ermöglicht eine anwendungsorientierte Bewertung unterschiedlicher RFID- 
Systeme. Durch die Kennzahlen Lesefähigkeit, Schreibfähigkeit, Kommunikationsunfähigkeit und Orientierungsunempfindlichkeit ist das Systemverhalten vollständig beschrieben. Zusammengefasst werden diese Kennzahlen durch die Kommunikationssicherheit. Durch die Betrachtung der benötigten Sendeleistung ist es erstmals möglich die Kennzahlen als Funktion der Sendeleistung darzustellen. Auf Basis der Sendeleistung ist es nun möglich jedes RFID-System hinsichtlich optimaler Sendeleistungsbedingungen zu bewerten. Die neu definierte Kommunikationsbelastbarkeit stellt die finale Kennzahl dar und ermöglicht eine komprimierte und dabei aussagekräftige Darstellung aller relevanten Ergebnisse. Anhand der Versuche konnte nachgewiesen werden, dass die Bewertung eines RFID-Systems nur mittels der Kommunikationsbelastbarkeit ausreichend ist. Für den Anwender reicht somit diese Information aus, um eine Bewertung unterschiedlicher RFID-System durchführen zu können.

Anwendung findet das Kennzahlensystem in dem am Institut für Transport- und Automatisierungstechnik der
Leibniz Universität Hannover befindlichen RFID-Prüfstand. Dieser ermöglicht es, Messungen für unterschiedliche Identifikationsszenarien automatisiert durchzuführen. Somit kann eine schnelle und genaue Bewertung verschiedener RFID-Systeme durchgeführt werden.

\section{FÖRDERHINWEIS}

Das IGF-Vorhaben 16833 N/1 der Forschungsvereinigung Forschungsgemeinschaft Intralogistik / Fördertechnik und Logistiksysteme e.V. (FG IFL), Lyoner Straße 18, 60528 Frankfurt am Main wird über die AiF im Rahmen des Programms zur Förderung der industriellen Gemeinschaftsforschung und -entwicklung (IGF) vom Bundesministerium für Wirtschaft und Technologie aufgrund eines Beschlusses des Deutschen Bundestages gefördert.

\section{LITERATUR}

[Deierling.2010]

A. Deierling, H. Ertl, J.-F. Höfinghoff, A. Jungk, D. Neuhäuser, C. Nitschke, S. Spengler, M. von Werder, L. Overmeyer, "Abschlussbericht: IdentProLog- Flexible Zielführung in Produktion und Materialflusslogistik durch vollständig in den Informationsfluss integrierte Flurförderzeuge", Leibniz Universität Hannover, PZH-Verlag, 2010.

[Höfinghoff.2011]

J.-F. Höfinghoff, A. Jungk, W. Knop, L. Overmeyer, "Using 3D Field Simulation for Evaluating UHF RFID Systems on Forklift Trucks", IEEE Transaction on Antennas and Propagation 59, Nr.2, 2011.

[Jungk.2007]

A. Jungk, G. Heiserich, L. Overmeyer, "Forklift Trucks as Mobile Radio Frequency Identification Antenna Gates in Material Flow", IEEE Intelligent Transportation Systems Conference, 2007.

[Jungk.2010]

A. Jungk, "Kennzahlbasierte Bestimmung der Leistungsfähigkeit von RFIDKomponenten am Flurförderzeug", Berichte aus dem ITA Bd.1., Leibniz Universität Hannover, PZH-Verlag, 2010.

[Nikitin.2006]

P. V. Nikitin, K. V. S. Rao, "Performance Limitations of Passive UHF RFID Systems", IEEE Antennas and Propagation Society International Symposium, 2006.

[Strauß.2009]

W. Strauß S. Kraus, M. Hartmann, C. Grabowski, J. Bernhard, "Read Range Measurements of UHF RFID Transponder in Mobile Anechoic Chamber", IEEE International Conference on RFID, 2009. 